ORIGINAL ARTICLE

\title{
Microevolution of Renibacterium salmoninarum: evidence for intercontinental dissemination associated with fish movements
}

\author{
Ola Brynildsrud ${ }^{1}$, Edward J Feil ${ }^{2}$, Jon Bohlin ${ }^{1}$, Santiago Castillo-Ramirez ${ }^{2}$, \\ Duncan Colquhoun ${ }^{3}$, Una McCarthy ${ }^{4}$, Iveta M Matejusova ${ }^{4}$, Linda D Rhodes ${ }^{5}$, \\ Gregory D Wiens ${ }^{6}$ and David W Verner-Jeffreys ${ }^{7}$ \\ ${ }^{1}$ EpiCentre, Department of Food Safety and Infection Biology, Norwegian School of Veterinary Science, Oslo, \\ Norway; ${ }^{2}$ Department of Biology and Biochemistry, University of Bath, Bath, UK; ${ }^{3}$ Section for Bacteriology, \\ Department of Laboratory Services, Norwegian Veterinary Institute, Oslo, Norway; ${ }^{4}$ Marine Scotland Science, \\ Aberdeen, Scotland, UK; ${ }^{5}$ NOAA, Northwest Fisheries Science Center, Seattle, WA, USA; ${ }^{6}$ USDA, National \\ Centre for Cool and Coldwater Aquaculture, Leetown, WV, USA and ${ }^{7}$ Cefas Weymouth Laboratory, The Nothe, \\ Weymouth, UK
}

\begin{abstract}
Renibacterium salmoninarum is the causative agent of bacterial kidney disease, a major pathogen of salmonid fish species worldwide. Very low levels of intra-species genetic diversity have hampered efforts to understand the transmission dynamics and recent evolutionary history of this Gram-positive bacterium. We exploited recent advances in the next-generation sequencing technology to generate genome-wide single-nucleotide polymorphism (SNP) data from 68 diverse $R$. salmoninarum isolates representing broad geographical and temporal ranges and different host species. Phylogenetic analysis robustly delineated two lineages (lineage 1 and lineage 2); futhermore, dating analysis estimated that the time to the most recent ancestor of all the isolates is 1239 years ago (95\% credible interval (Cl) 444-2720 years ago). Our data reveal the intercontinental spread of lineage 1 over the last century, concurrent with anthropogenic movement of live fish, feed and ova for aquaculture purposes and stocking of recreational fisheries, whilst lineage 2 appears to have been endemic in wild Eastern Atlantic salmonid stocks before commercial activity. The high resolution of the SNP-based analyses allowed us to separate closely related isolates linked to neighboring fish farms, indicating that they formed part of single outbreaks. We were able to demonstrate that the main lineage 1 subgroup of $R$. salmoninarum isolated from Norway and the UK likely represent an introduction to these areas $\sim 40$ years ago. This study demonstrates the promise of this technology for analysis of micro and medium scale evolutionary relationships in veterinary and environmental microorganisms, as well as human pathogens.

The ISME Journal (2014) 8, 746-756; doi:10.1038/ismej.2013.186; published online 31 October 2013

Subject Category: Microbial population and community ecology

Keywords: fish pathogens; microbial evolution; next-generation sequencing; Renibacterium salmoninarum; transmission history
\end{abstract}

\section{Introduction}

The causative agent of bacterial kidney disease (BKD) in salmonids, Renibacterium salmoninarum, is a Gram-positive slow-growing facultative intracellular pathogen. BKD, a chronic, progressive granulomatous infection, is a major threat to both farmed and wild salmonid fish species worldwide (Fryer and Sanders, 1981; Evelyn, 1993; Evenden et al., 1993; Fryer and Lannan, 1993; Wiens, 2011).

Correspondence: DW Verner-Jeffreys, Cefas Weymouth Laboratory, CEFAS, The Nothe, Barrack Rd, Weymouth DT4 8UB, UK.

E-mail: david.verner-jeffreys@cefas.co.uk

Received 5 June 2013; revised 4 September 2013; accepted 9 September 2013; published online 31 October 2013
It was first reported in the wild Atlantic salmon (Salmo salar L.) from rivers in Scotland and in brook and brown trout from the East coast of US in the 1930s (Earp et al., 1953; Smith, 1964).

The genome of $R$. salmoninarum consists of a single circular 3.15-Mbp chromosome with no known plasmids or phage elements (Wiens et al., 2008). As with other specialized intracellular pathogens, there is evidence of genome reduction (Wiens et al., 2008), and it has evolved mechanisms to evade detection by the host immune system (Grayson et al., 2002). R. salmoninarum survives, and possibly also replicates, within the macrophages of the kidney (Young and Chapman, 1978; Gutenberger et al., 1997). The bacterium is able to spread horizontally between fish hosts as well as vertically 
via the ova (Evelyn et al., 1986). Overt symptoms may not be seen until several months post infection, thus providing ample opportunity for horizontal transmission through stocks. Transovarial transmission additionally provides a mechanism for global dissemination of $R$. salmoninarum via commercial activity. These factors, coupled with a lack of efficient therapy and vaccine regimens for this disease (Elliott et al., 1989), have, in some countries, resulted in the imposition of movement controls on premises confirmed as positive for BKD, or the destruction of infected animals, disinfection and fallowing of premises (Richards, 2011).

There is continued speculation relating to the likely origins and spread of BKD. It is frequently detected in wild and hatchery-bred Pacific salmon (Oncorhynchus spp.) in both freshwater and oceanic phases (Banner et al., 1986; Kent et al., 1998; Meyers et al., 2003; Arkoosh et al., 2004). Paterson et al. (1979) also detected $R$. salmoninarum in the kidneys of Atlantic salmon returning to rivers in Eastern Canada using an indirect fluorescent-antibody technique. However, the significance of reservoirs of infection in wild and feral salmonid populations in Western Europe is unclear. A PCR-based survey of wild fish from six rivers in England and Wales reported an infection prevalence of $8 \%$ in grayling (Thymallus thymallus) and $4.8 \%$ in Atlantic salmon (Chambers et al., 2008). It has also not been determined whether the original outbreaks of the 'Dee Disease' in Scottish rivers (Smith, 1964) were caused by introduction of the pathogen from elsewhere (for example, via ova imported from North America), or the represented reoccurrence of a disease that had long been endemic in European populations of Atlantic salmon. In the UK there has also been debate as to the extent to which farmed rainbow trout infected with $R$. salmoninarum pose a risk to farmed (and wild) Atlantic salmon and the best ways to control these potential risks (Murray et al., 2011; Richards, 2011; Murray et al., 2012).

Highly discriminatory $R$. salmoninarum genotyping tools are required to address these questions. Several different molecular typing methods have been developed for $R$. salmoninarum (Grayson et al., 1999, 2000; Rhodes et al., 2000; Alexander et al., 2001). The data generated by these studies point to $R$. salmoninarum being highly clonal with very limited phenotypic and genetic variation. There is, therefore, a need for more powerful methods to resolve sub-lineages within the population and, in doing so, to reconstruct recent evolutionary history and patterns of transmission. To this end, we generated genome-wide single-nucleotide polymorphism (SNP) data using a next-generation sequence platform for 68 strains of $R$. salmoninarum isolated from different host species, and wide temporal and geographical ranges. The data were analyzed using methods pioneered for use with important human pathogens (for example, Harris et al., 2010; Mutreja et al., 2011; Holt et al., 2012). To our knowledge, this study represents the first application of this technology to a strictly animal pathogen.

\section{Materials and methods}

Bacterial strains and growth conditions

The sample was composed as follows: two of the original isolations from wild Atlantic salmon from the River Dee in 1960, 12 isolates from Norway, 7 isolates from New Brunswick on the East Coast of Canada, 11 isolates from the West Coast of the USA and Canada (including Washington, Oregon and British Columbia), 22 isolates from Scotland, 12 isolates from England and Wales, one single isolate from an eastern brook trout from Alberta, Canada and one isolate from a grayling from Montana, USA. Full details of these isolates are given in Table 1.

Cultures were grown on KDM solid media (Evelyn, 1977) at $15^{\circ} \mathrm{C}$, with colonies isolated and grown for DNA extraction.

\section{DNA extraction and sequencing}

Each participating laboratory separately prepared DNA from the isolates they supplied for the study. In brief, DNA was extracted from freshly grown bacteria harvested directly from solid media, resuspended in $500 \mu \mathrm{l}$ sterile deionized water, and then centrifuged at $14000 \mathrm{~g}$ for $15 \mathrm{~min}$. Or, in other cases, cultures of $R$ salmoninarum were grown in KDM broth to an $\mathrm{OD}_{525}$ and centrifuged at $10000 \mathrm{~g}$ for $20 \mathrm{~min}$. In all cases, the resultant bacterial pellets were then resuspended in $1 \mathrm{ml} 10 \mathrm{~mm}$ Tris and the DNA extracted as described by Wiens et al., 2002. There were no noticeable differences in the DNA quality in preparations from either solid or broth media (data not shown).

All the isolates (Table 1) were submitted for whole-genome, paired-end sequencing to The Genome Analysis Centre, Norwich, UK. DNA quality and yield was first determined by fluorometry using a Qubit fluorometer (Invitrogen) with QUANTiT dsDNA assay (Broad Range). The samples were also assessed for RNA contamination using a Qubit fluorometer with QUANT-iT RNA assay (Invitrogen). DNA TruSeq libraries were constructed for each isolate and were run on the Illumina (San Diego, CA, USA) HiSeq 2000 platform in pools of up to 12 libraries per lane.

\section{DNA TruSeq library construction}

The Illumina TruSeq DNA Sample Preparation was used to prepare pooled-indexed paired-end libraries of genomic DNA for subsequent cluster generation (Illumina cBot) and DNA sequencing using the reagents provided in the Illumina TruSeq DNA Sample Preparation v2 Kit. The samples (starting material of $\sim 1 \mu \mathrm{g}$ of genomic DNA) were sheared using a sonicator (Covaris (Woburn, MA, USA), S2/ LE220) to fragment sizes in the range of 200-700 bp. 
Table 1 R. salmoninarum isolates used in the study

\begin{tabular}{|c|c|c|c|c|c|}
\hline Isolate & Geographic origin & Year & Source ${ }^{\mathrm{a}}$ & Alternative $I D$ & $\begin{array}{c}\text { GenBank/ EBI } \\
\text { accession number }\end{array}$ \\
\hline Rs 10 & New Brunswick, Canada & 2009 & Salmo salar (sw) & & ERR327945 \\
\hline Rs 2 & New Brunswick, Canada & 2005 & S. salar (sw) & & ERR327951 \\
\hline Rs 3 & New Brunswick, Canada & 2005 & S. salar (fw) & & ERR327947 \\
\hline Rs 4 & New Brunswick, Canada & 2006 & S. salar (sw) & & ERR327946 \\
\hline Rs 5 & New Brunswick, Canada & 2007 & S. salar (sw) & & ERR327950 \\
\hline Rs 6 & New Brunswick, Canada & 2007 & S. salar (sw) & & ERR327953 \\
\hline Rs 8 & New Brunswick, Canada & 2008 & S. salar (sw) & & ERR327944 \\
\hline BPS 91 & Nanaimo, BC, Canada & 1991 & Oncorhynchus gorbuscha & & ERR327952 \\
\hline BQ96 91-1 & Nanaimo, BC, Canada & 1996 & Oncorhynchus kisutch & & ERR327963 \\
\hline DR143 & Alberta, Canada & 1972 & Salvelinus fontinalis $(\mathrm{fw})^{\mathrm{b}}$ & & ERR327954 \\
\hline 5006 & Bella Bella, BC, Canada & 1996 & O. kisutch (sw) & 960046 & ERR327942 \\
\hline 5223 & Kvinnherad, Hordaland, Norway & 2005 & S. salar (sw) & $2005-50-579$ & ERR327964 \\
\hline 6553 & Hemne, Sør-Trøndelag, Norway & 2008 & S. salar (sw) & $2008-09-495$ & ERR327955 \\
\hline 6642 & Hemne, Sør-Trøndelag, Norway & 2008 & S. salar & 2008-06-633 & ERR327956 \\
\hline 6694 & Hemne, Sør-Trøndelag, Norway & 2008 & Oncorhynchus mykiss (sw) & & ERR327962 \\
\hline 6695 & Hemne, Sør-Trøndelag, Norway & 2008 & O. mykiss (sw) & 2008-06-631 & ERR327968 \\
\hline 6863 & Osterøy, Hordaland, Norway & 2009 & O. mykiss (sw) & & ERR327965 \\
\hline 7439 & Sognefjorden, Sogn og Fjordane, Norway & 1984 & S. salar & 1984-40.992 & ERR327971 \\
\hline 7441 & Storfjord, Møre og Romsdal, Norway & 1985 & S. salar & $1985-09-667$ & ERR327966 \\
\hline 7448 & Stranda, Møre og Romsdal, Norway & 1986 & S. salar & 1986-09-4366 & ERR327970 \\
\hline 7449 & Skjervøy, Troms, Norway & 1987 & S. salar & 1987-09-932 & ERR327969 \\
\hline 7450 & Askøy, Hordaland, Norway & 1987 & S. salar & 1987-09-1185 & ERR327967 \\
\hline 684 & Aurland, Sognefjorden, Norway & 1987 & S. trutta (fw) & & ERR327958 \\
\hline 1205 & UK & 2001 & O. mykiss & $3104-67$ & ERR327930 \\
\hline 5007 & Scotland & 2005 & O. mykiss & $0180-18$ & ERR327923 \\
\hline 7105 & UK & 2007 & O. mykiss (fw) & P0416 T83 10-3 2 & ERR327932 \\
\hline 9025 & Yorkshire, England, UK & 2009 & O. mykiss (fw) & $16251-1$ & ERR327912 \\
\hline 96071 & England, Hampshire, site Z, UK & 1996 & O. mykiss (fw) & & ERR327927 \\
\hline 99326 & Wales, site Y, UK & 1999 & O. mykiss (fw) & $2119-8$ & ERR327938 \\
\hline 99327 & UK & 1997 & O. mykiss (fw) & $970313-2$ & ERR327931 \\
\hline 99329 & Wales, site $\mathrm{X}, \mathrm{UK}$ & 1998 & O. mykiss (fw) & $980036-125$ & ERR327937 \\
\hline 99332 & Wales, site Y, UK & 1999 & O. mykiss (fw) & 2119-3 & ERR327943 \\
\hline 99333 & Wales, site X, UK & 1998 & O. mykiss (fw) & 980036-102 & ERR327921 \\
\hline 99341 & Hampshire, site Z, England, UK & 1998 & O. mykiss (fw) & $980109-20$ & ERR327949 \\
\hline 99344 & Hampshire, England, UK & 1998 & O. mykiss (fw) & $980106-1.1 .5$ & ERR327940 \\
\hline 99345 & Wales, site X & 1998 & O. mykiss (fw) & $980070-18$ & ERR327948 \\
\hline NCIMB 1114 & River Dee, Scotland, UK & 1962 & S. salar $(\mathrm{fw})^{\mathrm{b}}$ & 5005 & ERR327908 \\
\hline NCIMB 1116 & River Dee, Scotland, UK & 1962 & S. salar $(\mathrm{fw})^{\mathrm{b}}$ & 96056 & ERR327907 \\
\hline MT239 & Scotland, UK & 1988 & S. salar & & ERR327913 \\
\hline MT1363 & Strathclyde, Scotland, UK & 1993 & O. mykiss (sw) & & ERR327920 \\
\hline MT3277 & Dumfries and Galloway Site A, Scotland,UK & 2008 & O. mykiss (fw) & & ERR327926 \\
\hline MT3313 & Central, Scotland, UK & 2008 & O. mykiss (fw) & & ERR327925 \\
\hline MT3315 & Strathclyde Site B, Scotland, UK & 2008 & O. mykiss (fw) & & ERR327928 \\
\hline MT1262 & Highlands, Scotland, UK & 1992 & S. salar (fw) & & ERR327922 \\
\hline MT1351 & Highlands, Scotland, UK & 1993 & S. salar (sw) & & ERR327904 \\
\hline MT1470 & Tayside, Scotland, UK & 1994 & O. mykiss (fw) & & ERR327910 \\
\hline MT1511 & Strathclyde Site B, Scotland, UK & 1994 & O. mykiss (fw) & & ERR327914 \\
\hline MT1880 & Strathclyde, Scotland, UK & 1996 & S. salar (sw) & & ERR327909 \\
\hline MT2622 & Strathclyde, Scotland, UK & 2002 & O. mykiss (sw) & & ERR327929 \\
\hline MT2943 & Highlands, Scotland, UK & 2005 & S. salar (sw) & & ERR327936 \\
\hline MT2979 & Highlands, Scotland, UK & 2005 & O. mykiss (fw) & & ERR327935 \\
\hline MT3106 & Strathclyde, Scotland, UK & 2006 & O. mykiss (fw) & & ERR327939 \\
\hline MT3479 & Orkney, Scotland, UK & 2008 & S. salar (sw) & & ERR327933 \\
\hline MT3482 & Strathclyde, Scotland, UK & 2009 & S. salar (sw) & & ERR327934 \\
\hline MT3483 & Strathclyde, Scotland, UK & 2009 & S. salar (sw) & & ERR327941 \\
\hline MT444 & Western Isles, Scotland, UK & 1988 & S. salar (sw) & & ERR327916 \\
\hline MT452 & Dumfries and Galloway Site A, Scotland,UK & 1988 & O. mykiss (fw) & & ERR327918 \\
\hline MT839 & Highlands, Scotland, UK & 1990 & S. salar (sw) & & ERR327917 \\
\hline MT861 & Scotland & 1990 & S. salar (sw) & & ERR327919 \\
\hline Car 96 & Washington State, USA & 1996 & O. tshawytscha & & ERR327957 \\
\hline D6 & Oregon, USA & 1982 & O. tshawytscha & & ERR327961 \\
\hline GR5 & Montana, USA & 1997 & T. thymallus $(\mathrm{fw})^{\mathrm{b}}$ & $980036-87$ & ERR327959 \\
\hline WR99 c2 & Washington State, USA & 1999 & O. kisutch & & ERR327960 \\
\hline NCIMB 2235 & Oregon, USA & 1974 & O. tshawytscha (sw) & ATCC33209 & ERR327911 \\
\hline $05372 \mathrm{~K}$ & Grande Ronde Basin, Oregon, USA & 2005 & O. tshawytscha (sw) & & ERR327906 \\
\hline Carson $5 b$ & Confluence Tyee Creek \& Wind River, WA, USA & 1994 & O. tshawytscha (fw) & & ERR327905 \\
\hline Cow-chs-94 & Cowlitz River, Washington & 1994 & O. tshawytscha (fw) & & ERR327915 \\
\hline ATCC $33209^{c}$ & Oregon, USA & 1974 & O. tshawvtscha (sw) & & NC 010168.1 \\
\hline NCIMB $1111^{\mathrm{d}}$ & - & Not known & Not known & 5004 & ERR327924 \\
\hline
\end{tabular}

Abbreviations: fw, fresh water; sw, sea water.

The complete history for some of the isolates is not known.

asolates recovered from fw or sw, where known.

'Isolate recovered from a wild fish species, all other isolates were recovered from farmed fish or not known.

'Used as a reference in this study. Sequence data downloaded from Genbank.

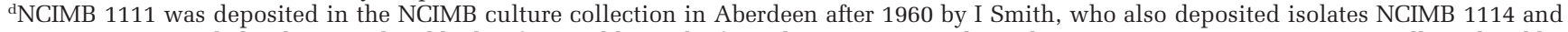
NCIMB 1116 recorded as being isolated by her from wild S. salar from the River Dee in the early 1960 s. NCIMB 1111 was reportedly isolated by Ken Wolf. Ken Wolf was a highly active US fish disease researcher who worked on US and Canadian strains of the pathogen in the 1950s and 1960s. It thus appears most likely that this isolate was a North American strain provided by Ken Wolf to I Smith to assist her with her studies on Dee disease (although this cannot be proven). 
The ends of the DNA were then repaired and a single A base added to each $3^{\prime}$ end of the DNA fragment to which an indexed adapter binds. A gel size selection method was used (Invitrogen E-Gel, 2\% agarose) to select the appropriate sized library that was then enriched by PCR, quantified using an Agilent (Santa Clara, CA, USA) DNA 1000 chip on the Agilent Bioanalyzer 2100, pooled with up to 12 other libraries and sequenced. Library preparation was automated using a Perkin Elmer (Waltham, MA, USA) (formally Caliper) Sciclone NGS Workstation (sonication, size selection, enrichment and pooling are not performed on the Sciclone).

\section{Data processing, genome alignment and assembly}

Close to 1000000000 pairs of reads, each of length $100 \mathrm{bp}$, were created for the project in total. The raw data have been deposited in the database of the European Bioinformatics Institute, and is available at http://www.ebi.ac.uk. Accession numbers are listed in Table 1. Reads were pre-filtered through the eliminate_singletons.py, eliminate_n_paired.py and filter_reads.py scripts from the BioPython (version 1.60) package (Cock et al., 2009) before assembly, excluding reads that would not properly pair, reads with ambiguous ('N') base calls and reads with an average PHRED quality score below 20.

We used MAQ v.0.7.1 (Li et al., 2008) to align raw reads to ATCC33209, the reference genome published in GenBank (http://www.ncbi.nlm.nih. gov/genbank. NCBI accession number: NC 010168.1). MAQs sol2sanger script was used to transform PHRED scores to the PHRED + 33 style. The vast majority of the reads mapped onto the reference genome, providing an average read depth across non-gap regions of 862.0 (Inter-isolates range: 57.2-2012.5). Statistics related to read output, read depth and read mapping is presented in Supplementary File SF3. Reads that mapped equally well to multiple areas of the reference genome, such as reads representing IS994 and ISRs2 sequences, were randomly assigned to one of the possible mapping locations. Variant calls from these areas were considered unreliable and were excluded from further analyses. Non-mapping reads were sorted into a separate file for separate de novo assembly in Velvet v.1.2.03 (Zerbino and Birney, 2008), using a k-mer length of 31. However, unmapped reads were all from Bacteriophage phi X 174, which was used as a positive control in the sequencing stage.

To validate these results, we also did de novo assembly using a combination of outputs from the DBG assemblers Velvet and ABySS (Simpson et al., 2009), as well as comparative assembly using the AMOScmp-shortreads tool from the AMOS package (Treangen et al., 2011). These assemblies were then merged using the minimus2 tool. This is an abridged version of the pipeline conceived and described by Ji et al., 2011. Comparative evaluation of the output from these two different pipelines was done in Hawkeye (Schatz et al., 2007). MAUVE (Darling et al., 2004) was used to screen for evidence of genomic rearrangements.

Variant calling and phylogeny reconstruction SNP calling was done with the default settings in MAQs cns2snp and maq.pl SNPfilter scripts. Furthermore, SNPs with PHRED quality scores of less than 255 were removed from analysis, as did SNPs with a high strand-bias. For a limited number of isolates, MAQ would occasionally produce ambiguous character SNP calls, even though PHRED qualities were consistently high. Closer inspection of the alignment revealed that the issue was caused by a low frequency $(<2 \%)$ of reads representing an alternative genotype. Although other plausible scenarios could explain this, slight DNA contamination is the most likely. We used the variants represented in the overwhelming majority of reads in these cases. Using SplitsTree4 (Graham et al., 2005), we could find no trace of recombination in our isolates. SNP sequences were then loaded into $\mathrm{R}$ (www.r-project.org) and processed using the ape (Paradis et al., 2004) and phangorn (Schliep, 2011) packages, in conjunction with the maximum-likelihood estimator PhyML (Guindon et al., 2010), to infer the optimal phylogenetic substitution model for the data. Several models were suggested as an outcome of this analysis, but the generalized timereversible model (Tavaré, 1986) without rate variation among sites obtained the lowest AIC score (Akaike, 1974), and was therefore chosen. Trees for both the full SNP alignment and pseudogene SNPs only were created with the MrBayes (Ronquist and Huelsenbeck, 2003) plugin in Geneious (version 6.0.3), using a generalized time-reversible-substitution model with a uniform site distribution. The Markov Chain Monte Carlo settings were set to include 10000000 generations with subsampling done every 2000th step. Burn-in was set to 1000000 generations, and the unconstrained branch length option was used. The tree was subsequently annotated in FigTree (http://www.tree.bio.ed.ac.uk/software/figtree/).

Bayesian Markov Chain Monte Carlo analyses A dated phylogeny was constructed by means of BEAST (Drummond and Rambaut, 2007; Drummond et al., 2012), based on the multiple alignment of all the SNPs that were not located in paralogous genes, using an uncorrelated lognormal relaxed clock with the generalized time-reversible model. Date of collection was used to estimate the divergence times of isolates. Two analyses were run for 600000000 generations, sampling every 30000 generations. We combined the results from the two independent runs through LogCombiner (excluding the first 10\% generations from each analysis). TRACER was used 
to evaluate the convergence of the combined analysis, the first $10 \%$ generations were discarded as burn-in; we corroborated that the effective sample size of all the parameters were greater than 200, and ensured that the trace plots of the likelihood scores randomly oscillated within a stable range. The mean rate of the molecular clock for the whole genome was calculated by multiplying the mean rate of the uncorrelated lognormal clock for the SNP collection by the SNP density in the genome.

\section{Ancestral character state reconstruction}

Mesquite (Maddison and Maddison, 2011) was used to reconstruct nodal character states based on terminal taxa values. Phylogenetic trees were constructed from pseudogene SNPs only. Both a parsimonious and a maximum-likelihood approach were used. For both approaches, geographical data were simplified to represent the country of origin in an unordered, character matrix. In the likelihood estimate, the one-parameter Markov k-state probability model (Lewis, 2001) was used.

\section{Accession numbers}

The sequence data for all the isolates were deposited in the European Bioinformatics Institute Short Read Archive under the accession numbers ERR327904 to ERR327971inclusive.

\section{Results}

\section{Phylogenetic analysis of $R$. salmoninarum}

A total of 3600 high-quality core-genome SNPs were identified (see Supplementary File 1), corresponding to one SNP every 876 bases. These were evenly distributed across the genome. There was no evidence of genomic rearrangement. Phylogenetic analysis resolved two major sub-lineages; lineage 1 and lineage 2 (Figure 1). Lineage 1 encompassed $90 \%$ of the studied isolates (61/68). These isolates were recovered from seven of the eight different host species, from the full range of geographical locations, and over a 50-year period (1960-2009). Despite this geographical, temporal and ecological diversity, lineage 1 isolates exhibit very low levels of
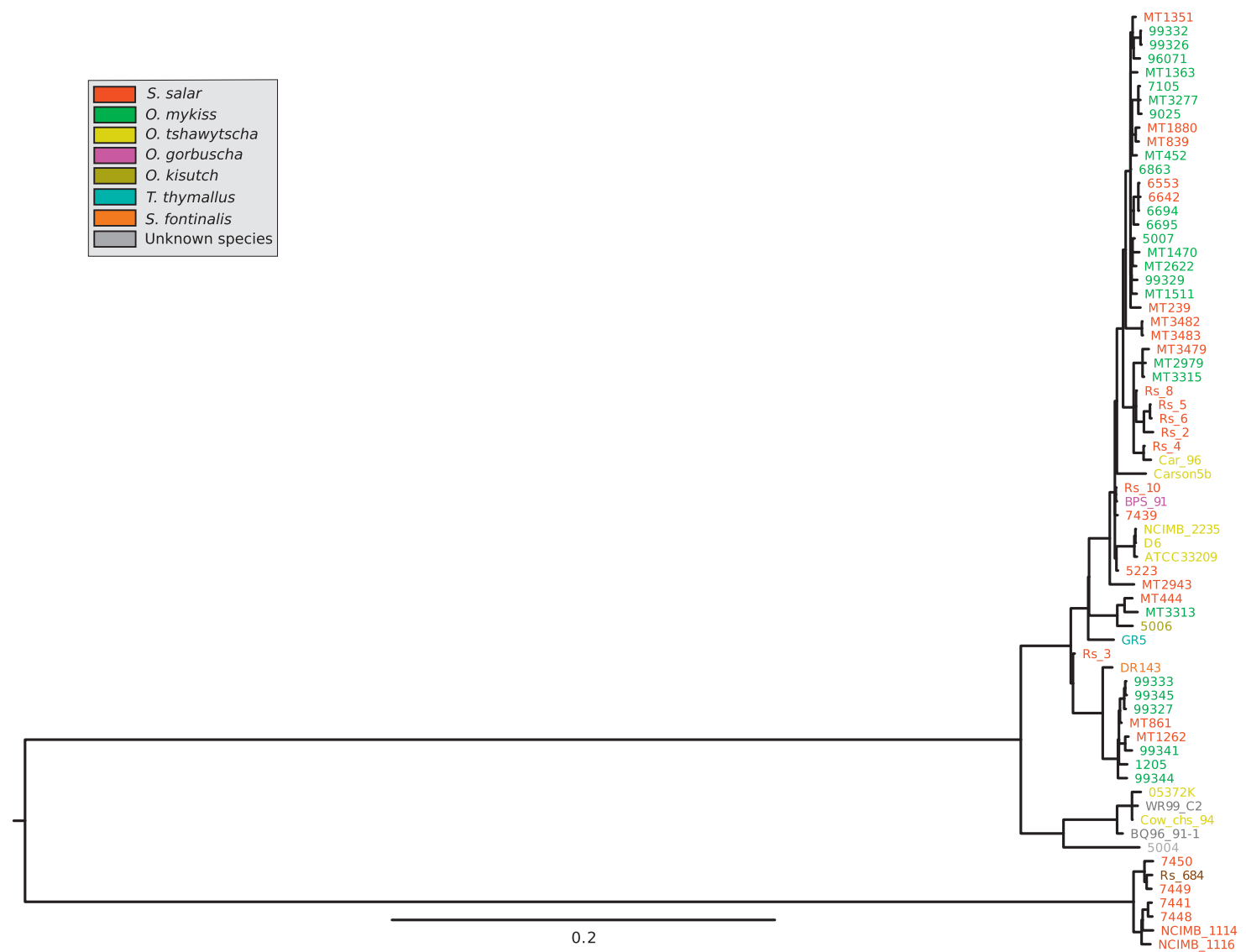

Figure 1 Phylogenetic tree of the 68 isolates of $R$. salmoninarum included in this study, showing all lineages. The evolutionary history was inferred using a Bayesian Markov Chain Monte Carlo approach, with a generalized time-reversible model (Tavaré, 1986), through the MrBayes (Ronquist and Huelsenbeck, 2003) plugin in Geneious. The consensus tree is taken to represent the evolutionary history of the taxa analyzed. The tree is drawn to scale, with branch lengths measured in the number of substitutions per site. All ambiguous positions were removed for each sequence pair. There were a total of 3600 positions in the final data set. The leftmost node represents a hypothetical most recent common ancestor. The above and bottom branches from this node represent lineages 1 and 2 , respectively. Isolates are color coded according to the host: green, rainbow trout; red, Atlantic salmon; yellow, Chinook salmon; pink, pink salmon; teal, Grayling; gold, Coho salmon; orange, Eastern brook trout; brown, brown trout; gray, not known. 
genetic diversity. The average pairwise nucleotide diversity $(\pi)$ across the whole genome $(3.15 \mathrm{Mb})$ within lineage 1 is 0.00005 , corresponding to an average of 167 SNP differences between pairs of strains. This paucity of variation is indicative of a slow rate of evolution, recent common ancestry of the population with global dissemination or both. The seven isolates of lineage 2 (including the isolates recovered from the River Dee in 1960), were all isolated from the UK and Norway and are exclusively associated with the genus Salmo; six from Salmo salar (Atlantic salmon) and one from Salmo trutta (brown trout). The average number of SNP differences between lineage 2 isolates is 80 , approximately half that of the lineage 1 isolates $(\pi=0.000025)$. The level of diversity between lineages is $\sim 20$-fold greater than the diversity within them, with an average inter-lineage difference of 2431 SNPs $(\pi=0.0008)$. The majority of the clades in the phylogeny are supported by high ( $>0.9$ ) PPS (posterior probability scores) (See Figure 2; all clades from linage 2 have complete support as evidenced by PPS of 1), indicating the phylogenetic analysis has not been significantly confounded by recombination. To confirm this, we checked for the presence of recombination using the
Phi test and splits decomposition as implemented in SplitsTree 4 (Graham et al., 2005). We carried out this analysis on the two major lineages separately, as the relatively large distance between them would impair visual inspection of the network. The Phi test did not find significant evidence for recombination either for Lineage $1(P=0.86)$ or for Lineage 2 $(P=0.1)$. Although inspection of the splits decomposition networks reveals very little reticulation for Lineage 1, slight reticulation is suggested between the seven strains of Lineage 2 (Supplementary Figure S1). The evidence thus points to a near absence of recombination, although a very limited degree of recombination cannot be excluded in Lineage 2.

\section{Inferring patterns of global transmission}

Lineage 1 contains a mixture of North American and European isolates, whilst lineage 2 is restricted to Europe. We used BEAST (Drummond and Rambaut, 2007; Drummond et al., 2012) for evolutionary analysis using an uncorrelated relaxed, lognormal clock. The mean clock rate was estimated as $3.324 \times 10^{-4}$ mutations/site/year for our 3600 SNPs, corresponding to an overall rate of $3.8 \times 10^{-7}$

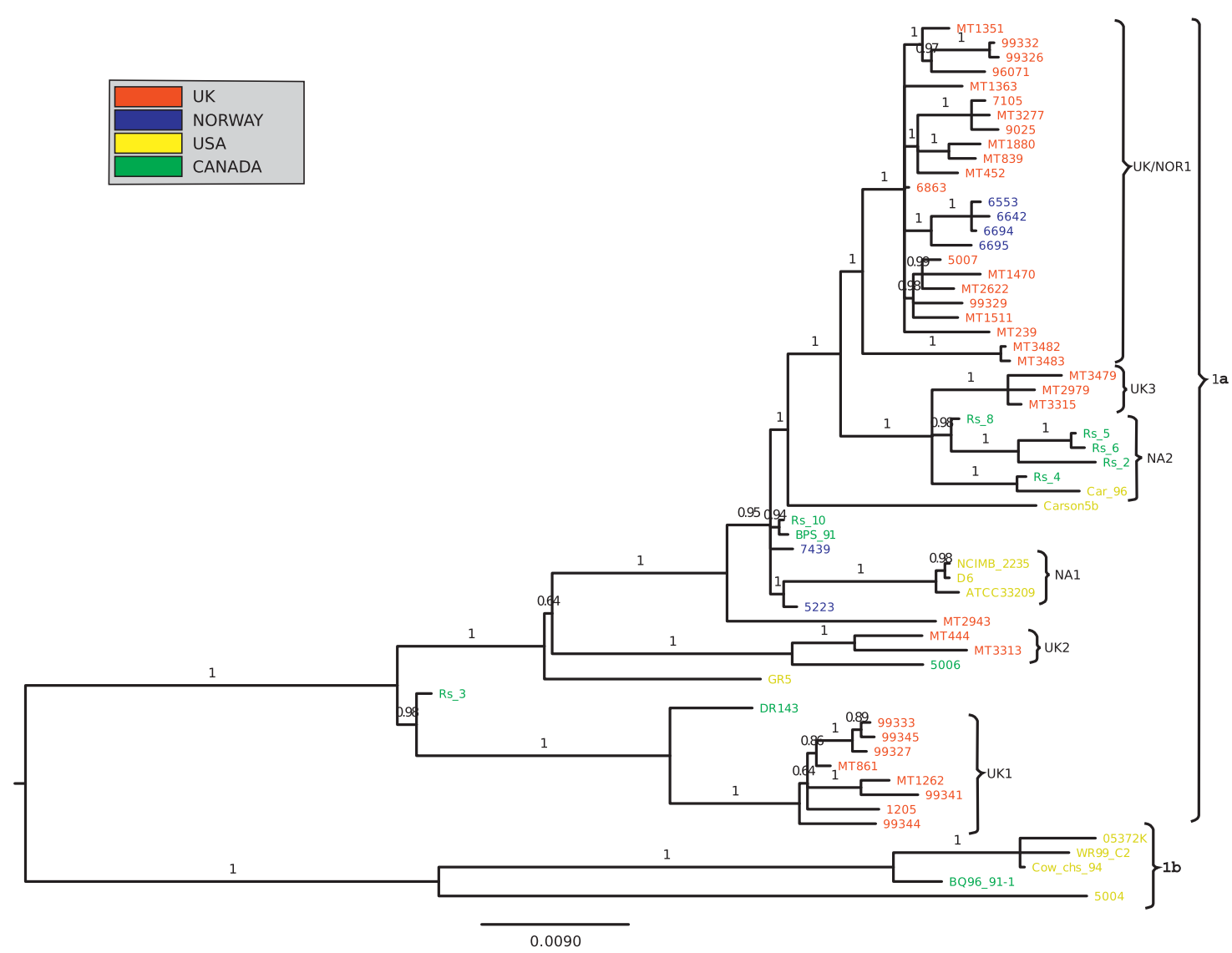

Figure 2 Detail of lineage 1. The phylogenetic tree was constructed as described under Figure 1. Posterior probability values are shown on each branch. For the detailed look at subgroup UK/NOR1, branches have been transformed so as to no longer represent evolutionary distance. Isolates are color coded according to their geographical origin: red, UK; blue, Norway; green, Canada; yellow, USA. 
mutations/genome/year. This figure represents the mean rate and may not be representative of individual lineages. The time to most recent common ancestor of all samples was dated to $\sim 1239$ years ago (95\% CI $444-2720$ years ago) (Supplementary Figure S2) However, it should be noted that the credible interval is rather wide leading to a not very precise point estimate; hence, caution should be exercised in considering this estimate. The lineages thus started to diverge at some point in time beyond this estimate. Lineage 1 can be further subdivided into lineage $1 \mathrm{a}$ and lineage $1 \mathrm{~b}$ (Figure 2). Lineage $1 \mathrm{~b}$ corresponds to five isolates from North America, consistent with a North American origin for this group. In contrast, Lineage 1a consists of isolates recovered from both North America and Europe. We note a general trend from the Lineage 1 tree that the most basal isolates tend to be of North American origin, supporting the view that this lineage emerged in North America and has more recently been transmitted to Europe rather than the other way around. For example, isolate Rs_3 was recovered from an Atlantic salmon in New Brunswick, Canada, and isolate DR143, which was isolated from a brook trout (Salvelinus fontinalis) from Alberta, Canada, both positioned in group 1a, basally to a cluster of eight isolates from the UK (labeled UK1 on Figure 2). The UK1 cluster may therefore represent a transmission event from Canada to the UK and, according to the BEAST analysis, this is most likely to have occurred $\sim 66$ years ago (95\% CI 40-120 years ago). The eight isolates corresponding to the UK1 cluster originate from fish farms in Wales, Scotland and England, indicating dissemination throughout UK farms subsequent to this transmission event that affected at least two commercial host species: Atlantic salmon (MT1262 and MT861) and rainbow trout (99333, 99327, 99341, 99344, 1205 and 99345). The large cluster of 22 isolates from the UK and Norway (UK/NOR1) is also consistent with a single recent introduction $\sim 43$ years ago (95\% CI 30-65 years ago.), followed by rapid spread between the UK and Norway, affecting multiple host species. Finally, two small clusters of UK isolates are evident in Lineage 1a (UK2, UK3), which may also represent independent introductions and transmission across multiple host species within the UK.

The inferences above are based largely on the casual inspection of the tree. In order to investigate the origins and transmission history of the $R$. salmoninarum isolates within a more robust framework, we performed ancestral state reconstruction using Mesquite (Maddison and Maddison, 2011). We used both parsimony (Figure 3) and maximum-likelihood (Supplementary Figure S3)based methods. In both cases, geographic origin was treated as a categorical variable with a uniform cost of switching. This parsimony analysis strongly supports the inferences discussed above in predicting a likely North American origin for the UK1, UK2, NA1, UK3 and NA2 groups. Although the immediate ancestor of the UK/NOR1 is predicted to originate from the UK (Figure 3; node A),

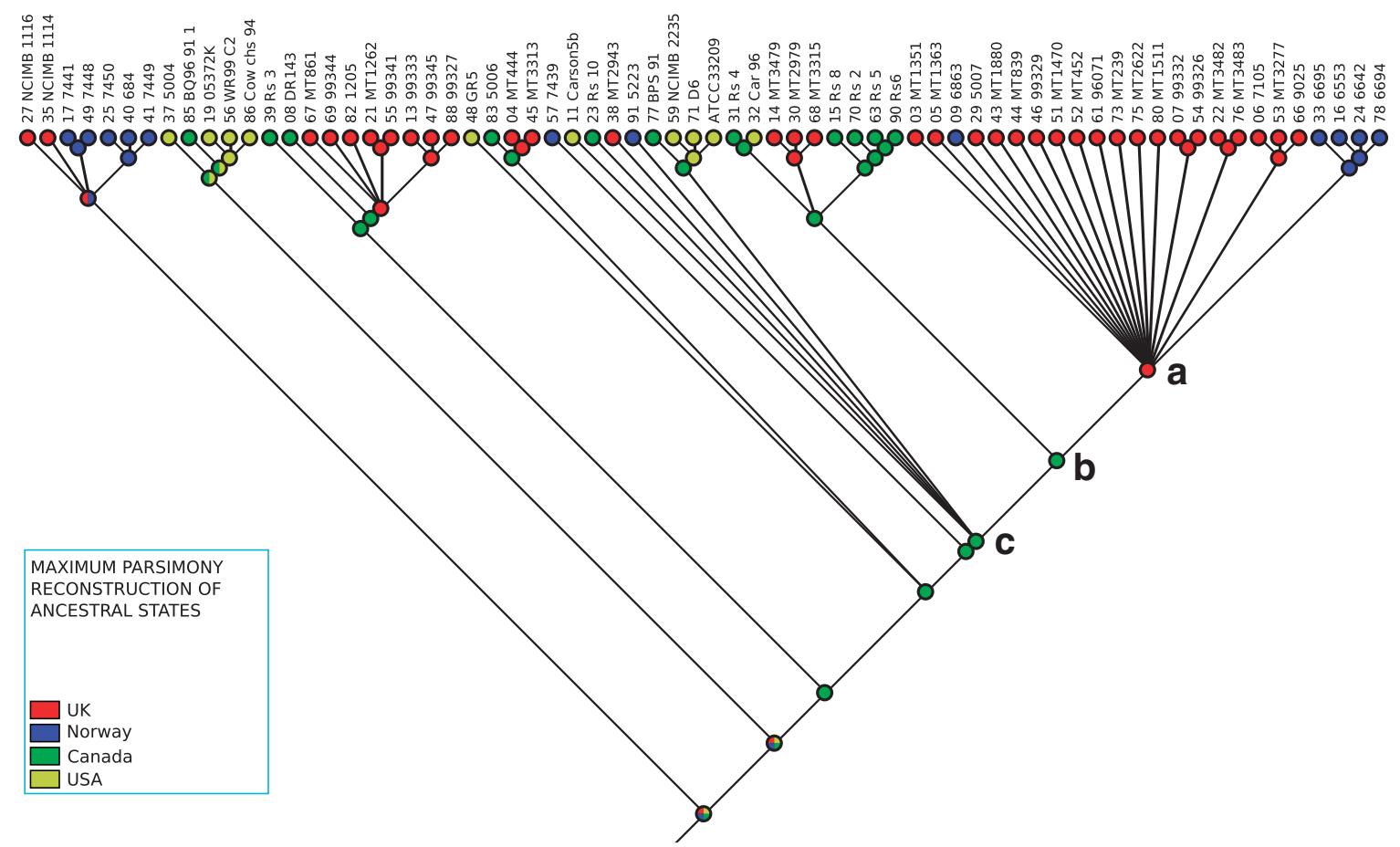

Figure 3 Ancestral state reconstruction of the geographical origin. Nodes in the trees have been estimated from a maximum parsimony evaluation of terminal values, where country of origin has been evaluated in an unordered, categorical matrix. The tree is not drawn to scale. The legend is as follows: red, UK; blue, Norway; green, Canada; yellow, USA. Nodes A, B and C are referenced in the text. The tree was calculated by using SNPs from open reading frames (ORFs) annotated as pseudogenes in the ATCC33209 genome. 
the analysis points to a character switch before the emergence of this clade as the most parsimonious states at nodes B and C are of Canadian origin. This character switch thus reflects a transmission from North America to the UK.

We also considered proportional likelihoods of each state at the different internal nodes in the tree as given in Supplementary File SF2. This maximum-likelihood approach unsurprisingly confirms that the immediate ancestor of the UK/NOR1 group (node A in Figure 3) was very likely of UK origin (node 52 in SF2; proportional likelihood score for UK origin $=1.0$ ). Moving one node back in the tree (node B in Figure 3) the proportional likelihoods of a UK and North American origin were found to be approximately equal (node 76 in SF2; proportional likelihood $=0.44$ for UK and 0.48 for Canada). However, moving back one node (node $\mathrm{C}$ in Figure 3) the maximum-likelihood analysis provides far stronger support for a North American origin, and very weak evidence for a UK origin (node 77 in SF2; proportional likelihood $=0.05$ for UK and 0.75 for Canada). This analysis therefore points to a switch (transmission) from North America to UK either between node $\mathrm{C}$ and node $\mathrm{B}$ (as suggested by maximum likelihood), or between node $B$ and node A (as suggested by maximum parsimony) (Figure 3).

\section{Detecting outbreaks}

In addition to providing evidence concerning largescale patterns of transmission, next-generation sequence data can also distinguish between samples of contemporaneous isolates recovered from a local setting. The technology allows the analyst to accurately assess whether or not concurrent presentations of disease form an outbreak or not. For instance, UK/NOR1 cluster isolates 6642, 6694 and 6553, recovered as part of disease outbreak investigations in the region of Sør-Trøndelag in 2008, were very closely related, differing at only five polymorphic sites. Strikingly, these three isolates also demonstrate free transmission between hosts; isolates 6642 and 6553 are from the same $S$. salar farm in Hemne, whereas 6694 and 6695 are from a nearby $O$. mykiss farm. In other cases the technology demonstrated that contemporaneous strains isolated from a single farm are not always epidemiologically linked, but may in some cases correspond to a mixture of the major lineages circulating throughout the UK and beyond. For example, isolates 99329, 99333 and 99345 were all isolated in 1998 from the same site in Wales but the latter two isolates belong to the cluster UK1, whilst 99323 belong to UK/NOR1.

\section{Discussion}

The very low density of polymorphisms and almost total conservation of gene content confirm that
$R$. salmoninarum is a highly clonal pathogen. A very low level of genome diversity has been noted in other highly specialized intracellular bacterial pathogens, notably Mycobacterium tuberculosis, which is phylogenetically related to $R$. salmoninarum and also causes a chronic granulomatous disease. Our estimate for a mean clock rate of $3.8 \times 10^{-7}$ mutations/bp/year is higher than a recent estimate for $M$. tuberculosis of $7.3 \times 10^{-8}$ (Ford et al., 2011. Scaled up from mutations/bp/day), and notably much higher than rates for Yersinia pestis that has been estimated at $8.6 \times 10^{-9}$ (Morelli et al., 2010). However, $R$. salmoninarum appears to mutate much more slowly than other major human pathogens such as Staphylococcus aureus, for which the rate was recently reported as $1.3 \times 10^{-6}$ (Holden et al., 2013).

Although isolates were recovered from many different species-Atlantic salmon, grayling, brook trout, rainbow trout and other Oncorhynchus spp.--they are almost indistinguishable genetically, indicating free interspecies transmission and broad virulence properties. Our data therefore suggest that previously observed differences in host susceptibility to BKD (Starliper et al., 1997) are likely to reflect host and/or environmental factors rather than variation within the pathogen. The limited genetic diversity of UK isolates in particular may be related to the controls implemented in response to the 1937 Diseases of Fish Act, which prohibited import of live salmonids into the UK and also made it illegal to import salmonid ova and any live freshwater fish species without a license (Hill, 1996). This has likely limited associated importation of pathogens such as $R$. salmoninarum, which could be one of the reasons that a relatively limited range of subtypes of this, and another important bacterial pathogen, Yersinia ruckeri (Davies, 1991; Wheeler et al., 2009) appear to be present within UK farmed fish. It has previously been noted that the main subtype of $Y$. ruckeri circulating in farmed UK rainbow trout also shows limited diversity and is different from the main strains circulating in Europe (Davies, 1991; Wheeler et al., 2009), suggesting these controls have restricted pathogen exchange between the UK and mainland Europe aquaculture production systems.

The data reveal evidence concerning long-term geographical structuring and global transmission. The construction of a high-resolution SNP-based phylogeny robustly resolved two major lineages of $R$. salmoninarum, lineage 1 and lineage 2 . Given the extremely low divergence within lineage 1 , it can be speculated that its rapid geographical and ecological dispersal, through different oceans and species of salmonids, has likely been facilitated by anthropogenic means. Commercial activities over the last 150 years have provided ample opportunities for such a spread to occur. Live salmonids and ova have been traded on a global scale for aquaculture, recreational angling and fishery stock enhancement purposes since the mid-19th century (Halverson, 2010). The 
last 40 years in particular have witnessed increasingly intensive and vertically integrated production, initially of rainbow trout and latterly of Atlantic salmon. Eggs are typically produced in dedicated broodstock facilities before being moved to hatcheries for production of fry, which are then moved again to ongrowing sites (either seawater cages for Atlantic salmon, or larger tanks or raceways for rainbow trout). As with other intensive livestock production systems, frequent transport means that breaches in biosecurity can lead to the transmission of pathogens between premises (Plarre et al., 2012). Furthermore, evidence exists that other highly clonal bacterial diseases of salmonids have spread between continents in similar ways, likely via egg and live fish movements. (Davies, 1991; Garcia et al., 2000; Wheeler et al., 2009).

In contrast to the global distribution of lineage 1, the absence of North American isolates in lineage 2 suggests that this lineage may represent a long-term endemic disease in European waters. Lineage 2 isolates all came from the North Sea area (including tributaries). The early outbreaks in Norway in the 1980s, all of lineage 2 origins, were reportedly in restocking hatcheries based on the capture and stripping of wild Atlantic salmon brood stock, and thus may have been caused by the pathogen present in wild populations. It is notable that the implementation of strict biosecurity measures and screening efforts in the late 1980s coincided with a significant reduction in outbreaks of BKD in Norway (Wiens and Dale, 2009; Johansen et al., 2011). The origin of lineage 1 is more unclear. The estimated phylogenetic division between the two main lineages ( 1000 years ago) clearly pre-dates commercial activity. It is possible that lineage 1 emerged independently within a geographically or ecologically isolated population of Atlantic salmonid before being transferred into Pacific salmonid populations. In support of this, BKD was not reported as a problem in Pacific salmon hatcheries on the West Coast of the US (Earp et al., 1953) until much later than East Coast hatcheries (Belding and Merrill, 1935). This is also consistent with the suggestion that the pathogen has co-evolved for longer with Atlantic salmonids (Salmo and Salvelinus), thus potentially explaining why Pacific salmon species are reportedly more susceptible to this pathogen (Evenden et al., 1993; Starliper et al., 1997). The relatively limited recovery of lineage 2 isolates as opposed to lineage 1 isolates in the UK and Norway was noteworthy. Further research is needed to establish whether any genetic advantage in either of the lineages can explain this asymmetry in distribution.

In general, the accuracy of determination of the relatedness of isolates demonstrated here was not possible with previously employed typing methods (Grayson et al., 1999, 2000; Rhodes et al., 2000). As an example, previous random amplified polymorphic DNA-based analysis (Grayson et al., 2000) grouped the DR143 and Cow chs 94 isolates on the same terminal branch, whereas our dated phylogenetic trees reveal that the most recent common ancestor of these isolates existed about 360 years ago. (95\% CI 150-700 years ago). This clearly illustrates the superiority of the next-generation sequence technology for outbreak determination problems.

Our data and analysis have delineated two major European clusters within Lineage 1a (UK1 and UK/NOR1) that emerged $<70$ years ago, probably as a result of transmission from North America, and have spread between fish farms in the UK and Norway infecting both rainbow trout and Atlantic salmon. Support for this comes from the observation that the North American lineage 1 isolates tend to be more diverse (and basal) than the European lineage 1 isolates, suggesting the former reflect the 'ancestral' population and the latter consist of independent introductions (bottlenecks). Our reconstruction of ancestral states analysis also points to the North American origin of most sub-lineages in Lineage 1. In summary, this points to transatlantic commercial activity as a likely factor in the European emergence and spread of lineage 1. However, it should be emphasized that all this refers only to the major European lineage 1 clades that we have sampled, and it is not possible to extrapolate from this to general conclusions about rates of transmission in either direction, and significant transmission from Europe to North America through commercial activity may also have taken place.

\section{Conclusion}

The application of whole-genome SNP-based comparisons has offered a range of insights into the likely microevolutionary relationships of this important fish pathogen that hitherto would not have been possible. The analysis reveals an unexpected deep phylogenetic division in the population, hinting at historical allopatry, evidence for transatlantic transmission and spread over the scale of decades and even proof of principle that the approach can be used to identify single outbreak strains on a very local scale. It is recommended that this methodology should also be applied for studies of other veterinary pathogens and environmental microorganisms, particularly those with very limited genetic intraspecies variation.

\section{Acknowledgements}

The UK's Department for Environment Food and Rural Affairs provided funding through Projects FB002 and an interagency grant DP258. Joel Caren, Inger Lise Engen and Georgina Rimmer provided valuable technical support. We thank Dr Bill Keleher for providing isolates from New Brunswick, Canada. Dr Chris Watkins from The Genome Analysis Centre (TGAC), Norwich, UK provided expert assistance with sequencing the isolates. We are grateful to the reviewers for their helpful comments. 


\section{References}

Akaike H. (1974). A new look at the statistical model identification. IEEE Trans Auto Contrl 19: 716-723.

Alexander SM, Grayson TH, Chambers EM, Cooper LF, Barker GA, Gilpin ML. (2001). Variation in the spacer regions separating tRNA genes in Renibacterium salmoninarum distinguishes recent clinical isolates from the same location. J Clin Microbiol 39: 119-128.

Arkoosh MR, Clemons E, Kagley AN, Stafford C, Glass AC, Jacobson K et al. (2004). Survey of pathogens in juvenile salmon Oncorhynchus Spp. migrating through Pacific Northwest estuaries. J Aquat Anim Health 16: 186-196.

Banner CR, Long JJ, Fryer JL, Rohovec JS. (1986). Occurrence of salmonid fish infected with Renibacterium salmoninarum in the Pacific Ocean. $J$ Fish Dis 9: 273-275.

Belding DL, Merrill B. (1935). A preliminary report upon a hatchery disease of the salmonidae. Trans Am Fish Soc 65: 76-84.

Chambers E, Gardiner R, Peeler EJ. (2008). An investigation into the prevalence of Renibacterium salmoninarum in farmed rainbow trout, Oncorhynchus mykiss (Walbaum), and wild fish populations in selected river catchments in England and Wales between 1998 and 2000. J Fish Dis 31: 89-96.

Cock PJA, Antao T, Chang JT, Chapman BA, Cox CJ, Dalke A et al. (2009). Biopython: freely available Python tools for computational molecular biology and bioinformatics. Bioinformatics 25: 1422-1423.

Darling ACE, Mau B, Blattner FR, Perna NT. (2004). Mauve: multiple alignment of conserved genomic sequence with rearrangements. Genome Res 14: 1394-1403.

Davies RL. (1991). Clonal analysis of Yersinia ruckeri based on biotypes, serotypes and outer membrane protein-types. J Fish Dis 14: 221-228.

Drummond A, Rambaut A. (2007). BEAST: Bayesian evolutionary analysis by sampling trees. BMC Evol Bio 7: 214

Drummond AJ, Suchard MA, Xie D, Rambaut A. (2012). Bayesian phylogenetics with BEAUti and the BEAST 1.7. Mol Biol Evol 29: 1969-1973.

Earp BJ, Ellis CH, Ordal EJ. (1953). Kidney disease in young salmon (No. 1). Special Report Ser. No. 1, Department of Fisheries, Washington State, USA, p 73.

Elliott DG, Pascho RJ, Bullock GL. (1989). Developments in the control of bacterial kidney disease of salmonid fishes. Dis Aquat Org 6: 201-215.

Evelyn TPT. (1977). An improved growth medium for the kidney disease bacterium and some notes on using the medium. Bull Off Int Epizoot 87: $511-513$

Evelyn TPT. (1993). Bacterial kidney disease-BKD. In: Inglis V, Roberts RJ, Bromage NR (eds) Bacterial Diseases of Fish. Blackwell Scientific Publications: Oxford, UK, pp 177-195.

Evelyn TPT, Prosperi-Porta L, Ketcheson JE. (1986). Experimental intra-ovum infection of salmonid eggs with Renibacterium salmoninarum and vertical transmission of the pathogen with such eggs despite their treatment with erythromycin. Dis Aquat Org 1: 197-202.

Evenden AJ, Grayson TH, Gilpin ML, Munn CB. (1993). Renibacterium salmoninarum and bacterial kidney disease-the unfinished jigsaw. Ann Rev Fish Dis 3: 87-104.

Ford CB, Lin PL, Chase MR, Shah RR, Iartchouk O, Galagan J et al. (2011). Use of whole-genome sequencing to estimate the mutation rate of Mycobacterium tuberculosis during latent infection. Nat Genet 43: 482-486.

Fryer JL, Lannan CN. (1993). The history and current status of Renibacterium salmoninarum, the causative agent of bacterial kidney disease in Pacific salmon. Fish Res 17: 15-33.

Fryer JL, Sanders JE. (1981). Bacterial kidney disease of salmonid fish. Ann Rev Microbiol 35: 273-298.

Garcia JA, Larsen JL, Dalsgaard I, Pedersen K. (2000). Pulsed-field gel electrophoresis analysis of Aeromonas salmonicida ssp. salmonicida. FEMS Microbiol Lett 190: $163-166$.

Graham J, McNeney B, Seillier-Moiseiwitsch F. (2005). Stepwise detection of recombination breakpoints in sequence alignments. Bioinformatics 21: 589-595.

Grayson TH, Atienzar FA, Alexander SM, Cooper LF, Gilpin ML. (2000). Molecular diversity of Renibacterium salmoninarum isolates determined by randomly amplified polymorphic DNA analysis. Appl Environ Microbiol 66: 435-438.

Grayson TH, Cooper LF, Atienzar FA, Knowles MR, Gilpin ML. (1999). Molecular differentiation of Renibacterium salmoninarum isolates from worldwide locations. Appl Environ Microbiol 65: 961-968.

Grayson TH, Cooper LF, Wrathmell AB, Roper J, Evenden AJ, Gilpin ML. (2002). Host responses to Renibacterium salmoninarum and specific components of the pathogen reveal the mechanisms of immune suppression and activation. Immunology 106: $273-283$.

Guindon S, Dufayard J-F, Lefort V, Anisimova M, Hordijk W, Gascuel O. (2010). New algorithms and methods to estimate maximum-likelihood phylogenies: assessing the performance of PhyML 3.0. Syst Biol 59: 307-321.

Gutenberger SK, Duimstra JR, Rohovec JS, Fryer JL. (1997). Intracellular survival of Renibacterium salmoninarum in trout mononuclear phagocytes. Dis Aquat Org 28: 93-106.

Halverson A. (2010). An Entirely Synthetic Fish: How Rainbow Trout Beguiled America and Overran the World. Yale University Press: New Haven, UK.

Harris SR, Feil EJ, Holden MTG, Quail MA, Nickerson EK, Chantratita N et al. (2010). Evolution of MRSA during hospital transmission and intercontinental spread. Science 327: 469-474.

Hill B. National legislation in Great Britain for the control of fish diseases (1996). Rev Sci Tech 15: 633-645.

Holden MTG, Hsu LY, Kurt K, Weinert LA, Mather AE, Harris SR et al. (2013). A genomic portrait of the emergence, evolution, and global spread of a methicillin-resistant Staphylococcus aureus pandemic. Genome Res 23: 653-664.

Holt KE, Baker S, Weill F-X, Holmes EC, Kitchen A, $\mathrm{Yu}$ J et al. (2012). Shigella sonnei genome sequencing and phylogenetic analysis indicate recent global dissemination from Europe. Nat Genet 44: 1056-1059.

Ji Y, Shi Y, Ding G, Li Y. (2011). A new strategy for better genome assembly from very short reads. $B M C$ Bioinformatics 12: 493.

Johansen LH, Jensen I, Mikkelsen H, Bjørn PA, Jansen PA, Bergh Ø. (2011). Disease interaction and pathogens 
exchange between wild and farmed fish populations with special reference to Norway. Aquaculture 315: 167-186.

Kent ML, Traxler GS, Kieser D, Richard J, Dawe SC, Shaw RW et al. (1998). Survey of salmonid pathogens in ocean-caught fishes in British Columbia, Canada. J Aquat Anim Health 10: 211-219.

Lewis PO. (2001). A likelihood approach to estimating phylogeny from discrete morphological character data. Syst Biol 50: 913-925.

Li H, Ruan J, Durbin R. (2008). Mapping short DNA sequencing reads and calling variants using mapping quality scores. Genome Res 18: 1851-1858.

Maddison WP, Maddison DR. (2011). Mesquite: a modular system for evolutionary analysis. Version 2.75 http://mesquiteproject.org.

Meyers TR, Korn D, Glass K, Burton T, Short S, Lipson K et al. (2003). Retrospective analysis of antigen prevalences of Renibacterium salmoninarum (Rs) detected by enzyme-linked immunosorbent assay in Alaskan Pacific salmon and trout from 1988 to 2000 and management of Rs in hatchery chinook and coho Salmon. J Aquat Anim Health 15: 101-110.

Morelli G, Song Y, Mazzoni CJ, Eppinger M, Philippe R, Wagner DM et al. (2010). Phylogenetic diversity and historical patterns of pandemic spread of Yersinia pestis. Nat Genet 42: 1140-1143.

Murray AG, Hall M, Munro LA, Wallace IS. (2011). Modelling management strategies for a disease including undetected sub-clinical infection: bacterial kidney disease in Scottish salmon and trout farms. Epidemics 3: 171-182.

Murray AG, Munro LA, Wallace IS, Allan CET, Peeler EJ, Thrush MA. (2012). Epidemiology of Renibacterium salmoninarum in Scotland and the potential for compartmentalised management of salmon and trout farming areas. Aquaculture 324-325: 1-13.

Mutreja A, Kim DW, Thomson NR, Connor TR, Lee JH, Kariuki S et al. (2011). Evidence for several waves of global transmission in the seventh cholera pandemic. Nature 477: 462-465.

Paradis E, Claude J, Strimmer K. (2004). APE: Analyses of Phylogenetics and Evolution in $\mathrm{R}$ language. Bioinformatics 20: 289-290.

Paterson WD, Gallant C, Desautels D, Marshall L. (1979). Detection of bacterial kidney disease in wild salmonids in the Margaree river system and adjacent waters using an indirect fluorescent antibody technique. J Fish Res Board Can 36: 1464-1468.

Plarre H, Nylund A, Karlsen M, Brevik Ø, Sæther PA, Vike S. (2012). Evolution of infectious salmon anaemia virus (ISA virus). Arch Virol 157: 2309-2326.

Rhodes LD, Grayson TH, Alexander SM, Strom MS. (2000). Description and characterization of IS994, a putative IS3 family insertion sequence from the salmon pathogen Renibacterium salmoninarum. Gene 244: 97-107.

Richards R. (2011). A strategy for the control of Bacterial Kidney Disease (BKD) in Great Britainhttp://www. scotland.gov.uk/Resource/Doc/1062/0114801.pdf.
Ronquist F, Huelsenbeck JP. (2003). MrBayes 3: Bayesian phylogenetic inference under mixed models. Bioinformatics 19: 1572-1574.

Schatz M, Phillippy A, Shneiderman B, Salzberg S. (2007). Hawkeye: an interactive visual analytics tool for genome assemblies. Genome Biol 8: R34.

Schliep KP. (2011). phangorn: phylogenetic analysis in R. Bioinformatics 27: 592-593.

Simpson JT, Wong K, Jackman SD, Schein JE, Jones SJM, Birol İ. (2009). ABySS: a parallel assembler for short read sequence data. Genome Res 19: 1117-1123.

Smith IW. (1964). The Occurrence and Pathology of Dee Disease. Freshwater and Salmon Fisheries Research, 34. Her Majesty's Stationery OfficeEdinburgh, UK.

Starliper CE, Smith DR, Shatzer T. (1997). Virulence of Renibacterium salmoninarum to salmonids. J Aquat Anim Health 9: 1-7.

Tavaré S. (1986). Some probabilistic and statistical problems in the analysis of DNA Sequences. Lect Math Life Sci 17: 57-86.

Treangen TJ, Sommer DD, Angly FE, Koren S, Pop M. (2011). Next generation sequence assembly with AMOS. Curr Protoc Bioinform 33: 11.8.1-11.8.18.

Wheeler RW, Davies RL, Dalsgaard I, Garcia J, Welch TJ, Wagley $S$ et al. (2009). Yersinia ruckeri biotype 2 isolates from mainland Europe and the UK likely represent different clonal groups. Dis Aquat Org 84: 25-33.

Wiens GD. (2011). Bacterial Kidney Disease (Renibacterium salmoninarum). Fish Diseases and Disorders: Volume 3: Viral, Bacterial and Fungal Infections 3, 2nd ednWoo PTK, Bruno DW (eds) 338-374.

Wiens GD, Dale OB. (2009). Renibacterium salmoninarum p57 antigenic variation is restricted in geographic distribution and correlated with genomic markers. Dis Aquat Org 83: 123.

Wiens GD, Pascho R, Winton JR. (2002). A single Ala139to-Glu substitution in the Renibacterium salmoninarum virulence-associated protein p57 results in antigenic variation and is associated with enhanced p57 binding to chinook salmon leukocytes. Appl Environ Microbiol 68: 3969-3977.

Wiens GD, Rockey DD, Wu Z, Chang J, Levy R, Crane $S$ et al. (2008). Genome sequence of the fish pathogen Renibacterium salmoninarum suggests reductive evolution away from an environmental Arthrobacter ancestor. J Bacteriol 190: 6970-6982.

Young CL, Chapman GB. (1978). Ultrastructural Aspects of the Causative Agent and Renal Histopathology of Bacterial Kidney Disease in Brook Trout (Salvelinus fontinalis). J Fish Res Board Can 35: 1234-1248.

Zerbino DR, Birney E. (2008). Velvet: Algorithms for de novo short read assembly using de Bruijn graphs. Genome Res 18: 821-829.

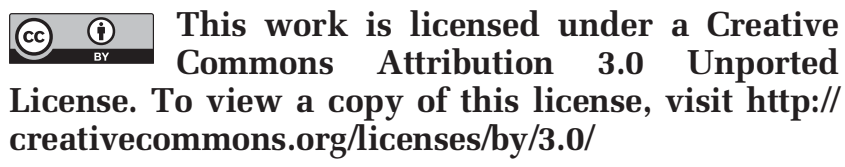

Supplementary Information accompanies this paper on The ISME Journal website (http://www.nature.com/ismej) 